\title{
A New Dispatch Control Integration System of the Smart Grid Based on the Regional Network Centralized Protected Mode
}

\author{
Zhang Peng, Liu Na, Chang Jing, Zhao Qi-feng, and Lin Man-man
}

\begin{abstract}
The hierarchical management and monitor model is a popular approach for the dispatch control mode of the smart grid. However, this model has many defects, e.g., the management of the data flow in the dispatch control system is very difficult, and the running efficiency of the dispatch control system is very bad too. This paper presents a regional network centralized protected mode(RNCPM) of the smart grid and establishes a new dispatch control integration system (DCIS)of the smart grid. The system architecture and key technologies of the DCIS based on RNCPM are proposed. A new communication architecture and the new features of the unified cross-section panoramic data(UCSPA) is studied. A new scheduling operation and management mode and panoramic data system platform of smart grid are developed. The DCIS under RNCPM can have integrated the schedule and monitor of the smart grid. The proposed system will lead to a new operation and maintenance mode of smart grid. The new system will further optimize the scheduling and workflow transformer of the substation. And the overall level of smart grid security and emergency levels will be significant raised.
\end{abstract}

Index Terms-Dispatch control integration, regional network centralized protection, smart grid, panoramic data.

\section{INTRODUCTION}

The hierarchical management and centralized monitor and model is a popular approach for the dispatch control mode of the smart grid [1]-[3], that is, using "Regional monitoring center + Operation \& maintenance station" management model. This model has the advantages of safety, rapid reaction, good continuity of operation mode and equipment. Although this dispatch control mode of the smart grid has played a great role in the efficient management and development of the power grid, it has brought some problems. Firstly, the information communication and Information sharing between scheduling system and monitoring system are difficult. Secondly, the separately configured control system and dispatching system of power grid should be

Manuscript received April 2, 2018; revised July 5, 2018. This research is supported by the Department of Education Fund Project of Henan Province, China (Project nos.12B510037 and 13B510296), by the Department of Science \&Technology Fund Project of Henan Province, China (Project nos. 162102210092 and 142102210579), and by the Zhengzhou Administration of Science \& Technology Fund Project of Henan Province, China (Project no. 141PPTGG363)

Zhang Peng is with the Zhongyuan University of Technology, Zhengzhou, 450007, China. He is now the HaMi Technical College, HaMi 100875, China (e-mail: zhangpengbbc@163.com).

Liu Na is with HaMi Technical College, HaMi 100875, China (e-mail: 779836544@qq.com).

Chang Jing, Zhao Qi-feng, and Lin Man-man are with the Zhongyuan University of Technology, Zhengzhou, 450007, China (e-mail 271014227@qq.com). separately set up and maintained, and the inconsistency of the separately configured control system and dispatching system will usually lead the difficulty of dealing with the accident to the regional monitoring center and Operation \& maintenance station. Thirdly, with the rapid development of the power grid, the number of centralized control center is increased rapidly too. Each independent centralized control center should be deployed with full set of operation and maintenance personnel, which cannot achieve the goal of reducing human resources and improving production efficiency. Fourth, With the gradual development of smart grid research and application, the traditional specialty division of the relay protection, communications, automation and other specialty according to the traditional operation and management mode has not adapted to the development of smart grid technology. The specialty must be integrated to achieve the intensive, standardized operation of the staff and management. Fifth, the degree of the intensive production organization and specialization of the traditional power grid is not high. The traditional power grid management chain is too long, and the resource allocation efficiency is very low. The traditional scheduling, operation management mode of the substation has been unable to adapt to the rapid development of smart grid.

RNCPM of the smart grid can solve the exiting problem of the power grid dispatch control. To solve the problem of the power grid dispatch control, a new DCIS based on RNCPM is proposed. In this mode, the comprehensive utilization of optical fiber communication technology, computer technology, sensing technology, relay protection and control technology, power grid operation technology and other technologies can significantly improve the dispatching performance of the power grid regulation and control. The IEC61850 standard [3], [4] is implemented and extent on the level of scheduling and controlling [5], [6]. By centralizing the configuration and management of the dispatching protection equipment within the scope of centralized dispatching and control, integration of dispatching automation and protection control is realized.

DCIS based on RNCPM highly fuses the control, scheduling and monitoring of the power grid. The integration system will lead to a great change of power grid operation and management mode, and will further optimize the power grid dispatch substation workflow, and improve the daily operating efficiency and the power grid accident treatment efficiency. Hence, this system will fully enhance power grid security and emergency level. Therefore, in this paper, it is of great significance to study the key technologies of the DCIS based on RNCPM. 
The remainder of this paper is organized as follows. In the next section, we survey the development and application of DCIS based on RNCPM. The proposed system technology architecture and key technologies are overviewed. Section IV presents the system communication architecture. Section V addressed the system panoramic data platform. New features of unified section panoramic data based on RNCPM are discussed too. Section VII reports analysis of dispatching operation management of DCIS based on RNCPM. Section VIII reports a series of advanced application of power grid intelligent dispatching based on RNCPM. The conclusions drawn from this paper are given in Section IX.

\section{DeVElopment AND APPliCAtion OF DCIS BASED ON RNCPM}

\section{A. Summary of Domestic Situation}

At present, the integration of regional network dispatch control in China's power grid is in the R \& D stage [7], [8], [9]. Integration of the centralized monitoring and control of substation has been carried out in Beijing, Fujian and other provinces in China. However, the integration of power grid regulation and control in these provinces is still in the pilot phase. The operation mode, management system, scheduling relations and other aspects of those integration of power grid dispatch control still need to be further improved.

Beijing Power Grid Corp established Beijing power grid dispatch control center in December 2007. The operators on duty from power grid dispatching system has amalgamated with those from power grid control system. In the early stage, the dispatching system and control system are in parallel setting, and the operators on duty from dispatching system work together with those from control system in the same office. With the development of work in the Beijing power grid dispatch control center, the complete integration of operators and work business from dispatching system and control system is gradually realized.

Fujian Provincial Electric Power Company has determined the integrated management mode of monitoring, dispatch control, and select Xiamen Electric Power Bureau, Quanzhou Power Bureau as a pilot unit in State Power Bureau, Shishi County Power Company as the company's pilot units. Fujian Provincial Electric Power Company started these experimental reforms from April 2009. The integrated management mode of monitoring, dispatch control of Xiamen Electric Power Bureau was officially put into operation in October 2009. In addition to the original scheduling control functions, the additional capabilities added in the dispatch control center of the Xiamen Electric Power Bureau consist of the daily operation monitoring of substation, integrated monitoring of security signals of video, firefighting and security in substation, the switch remote operation of the power grid accident isolation and power rationing, emergent power transfer system of 110 thousand volts and below etc. In addition to the operation monitoring of the substation, the original functions of the dispatch control center remain unchanged.

Based on the operation experience of the pilot project, a few provinces and cities in China have taken the lead in achieving the integration of power grid regulation and control.
The integration mode of regulation and control is more intuitive, more active and faster to ensure the safe operation of power grid, which can better cope with the challenges of various complex power grid operation environment. However, the integration of regulation and control based on regional network centralized protection is still in the theoretical stage, and the related research work needs further development.

\section{B. Summary of Foreign Situation}

The operation of power grid in developed countries basically adopts the mode of "dispatch control integration"[10]-[12]. Especially, Norway's power grid is the most typical. There are altogether 130 substations in Norway power grid, and all are unmanned management substations. The power grid operation department is responsible for power grid dispatching and substation control. Three regional control centers are responsible for controlling the substations in the north, middle and south of Norway power grid respectively. The regional control center is responsible for the operation monitoring, equipment condition monitoring and circuit breaker monitoring of the substation. The isolating knife switch and ground knife in the substation are all electric operation mechanisms and can be remote controlled. When the repair task needs to be removed, the operation can be completed in the control center, including the disconnection of the knife switch and the closing of the ground knife. At the same time, the maintenance personnel are sent to the scene to inspect and check before the maintenance work is carried out. However, there is no precedent for the DCIS based on RNCPM.

\section{The TeChNOLOgY ARChitecture AND Key TECHNOLOGIES OF THE DCIS BASED ON RNCPM}

\section{A. The Technology Architecture of the DCIS Based on RNCPM}

To achieve the integration of dispatching automation, protection and control, the DCIS based on RNCPM centralizes the protection equipment within the scope of dispatch. The schematic diagram of the DCIS based on RNCPM is shown in Fig. 1. In Fig. 1, it can be found that the system structure can be divided into three layers. The specific contents are as follows:

1) Process level (substation level): This layer consists of local deployed panoramic data processing units. The panoramic data processing units set merging unit and intelligent terminal function in one. And It can realize the functions of fast main protection, synchronous data measurement, protection of sampling value and measurement data.

2) Protection layer: The main and backup protection in the DCIS based on RNCPM are all concentrated in the protective device of the protection layer. To realize the transmission flow control of network sampling value and expand the CPU computing ability of protection, the protection device of protection layer is mostly realized by the protection cabinet.

3) Integrated design of power grid dispatch \& control and regional monitoring background can achieve the integration of power grid protection, monitoring and control

In Fig. 1, the following features can be found. The 
RNCPM is adopted in power grid. And the whole data and information uploaded to the dispatch control system must be the real-time three-state panorama data with synchronous time scales. It is of great significance to realize real-time on-line monitoring and security \& stability analysis of the power grid dispatch control. At the same time, due to the rapid expansion of the scale of power grid and the number of the intelligent substation, the amount of data and information uploaded to the dispatch control center based on RNCPM is huge. Hierarchy, classification and integrated management techniques of the data and information are needed to reduce the amount of data uploaded to the dispatch control center. It can significantly improve the operation, dispatch control efficiency of the grid.

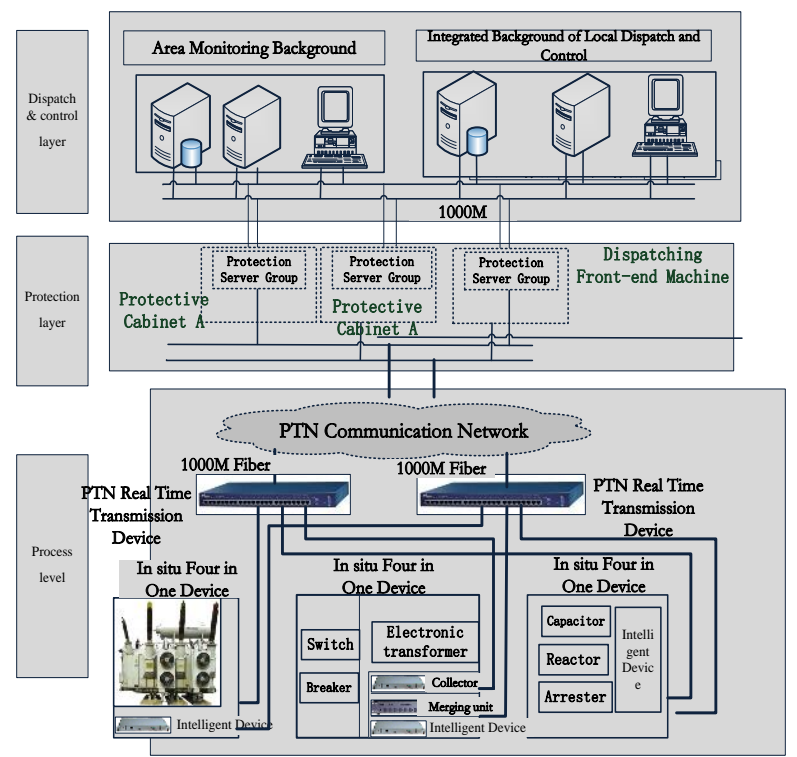

Fig. 1. The technology architecture of the DCIS based on RNCPM.

\section{B. The Key Technologies of the DCIS Based on RNCPM}

The key technologies of the DCIS based on RNCPM are as follows.

1) Basic supporting technology of the Integration system of power grid dispatch control

Dynamic data platform technology [13]-[15]. Dynamic data platform can provide analytical data for power grid scheduling and decision support systems. The dynamic data platform combines the offline data of all levels of power grid with the EMS (Energy Management System) online data resources. And the on-line operation data of power grid is introduced into the traditional stability analysis and calculation with the help of dynamic data platform technology. The advanced calculation analysis of power grid will be more in line with the actual operational aspect.

Parallel computing platform technology [16], [17]. Parallel computing platform is the computing carrier of scheduling and decision support system. The parallel computing platform is divided into online parallel computing platform and offline parallel computing platform.

The storage and management technology of historical data. The technology can effectively store and manage many periodic running data collected online. It is beneficial to offline research and use.

2) Online security analysis and decision-making technology
The functions of the power grid based on this technology include the safety and stability forewarning, dispatching assistant decision making, on line calculation of safety margin, monitoring and analysis of low frequency oscillation, energy plan security checking, calculation of large scale offline operation mode.

\section{3) Unified modeling technology for power grid model}

Unified modeling management platform of power grid model is a whole area unified model management system based on power grid model. It integrates various models of energy management system, power distribution management system and wide area measurement system. Through the distributed modeling and mosaic technology, the unified model of the whole area grid system is achieved with the goal of " Source end maintenance, whole grid sharing ". On this basis, the unified management of real-time, planned and other type of power grid application models is formed. Through the publishing service of the power grid model, it can meet the needs of the power grid model of various types of application of the dispatching center. And It will lay a solid foundation for the analysis, calculation, early warning and auxiliary decision of the dispatching center based on the whole power grid model.

4) Distributed state estimation technique for power grid

Distributed state estimation technique for power grid includes distributed state estimation technique, state estimation technique of master station, Integrated data state estimation technique for master station system based on PMU measurement, distributed state estimation technique of plant side, information interaction technology between main station and substation, information stitching technology of master station, etc.

\section{5) High reliability technology of power grid}

Integration system of power grid dispatch control uses a set of technologies to achieve system scheduling and centralized control \& monitoring operation, and the reliable operation of the system is vital to the safety of power grid. The research of high reliability technology of power grid needs to be explored in many aspects, such as the existing technology, combining with the actual situation, bold vision and rigorous testing. It can effectively prevent the potential operational risk of the DCIS based on RNCPM.

\section{The System COMMUnicAtion ARCHITECTURE}

The function of the Substation control layer is moved up to the regional monitoring background equipment of the control layer in the DCIS based on RNCPM. The main and backup protection in regional power grid are all concentrated in the protective device of the protection layer (i.e. equipment layer), (see Fig. 1). Integration system of power grid dispatch control is integrated with the regional monitoring station. At the same time, the process layer can realize the synchronous acquisition and upload function of the whole grid data. Therefore, the DCIS based on RNCPM will present the following advantages:

(1) Substation equipment on site. substation measurement $\&$ intelligent components and the primary equipment are integrated and installed. The main control room is cancelled in the substation, which greatly reduces the number of the 
substation secondary equipment and reduces the occupation area.

(2) Integration of the protection and monitoring. According to the dispatching management scope, the system functions of relay protection and substation automation are all moved to the dispatching control center. The system is upgraded from the traditional component oriented protection to the system oriented protection, which can meet the new demand of the new energy access, loop network operation, etc.

(3) Integration of dispatching and control. The integration system of power grid dispatch control is integrated with the substation automation system. And it can realize the integration of the protection, monitoring and control of the power grid.

(4) Intelligent operation of power grid. The intelligent monitoring, protection, control, measurement and operation $\&$ maintenance management of the power grid can be realized by using the panoramic real-time information.

(5) Transient stability analysis is carried out based on the synchronous real-time information of regional power grid.

The advantages of the DCIS based on RNCPM show that the communication architecture of the existing automatic control system cannot meet the new demands from the new power grid RNCPM. To meet the new demand, the DCIS based on RNCPM cancels the substation layer. In the substation process layer, the panoramic data acquisition of the primary equipment is carried out by intelligent integrated equipment. And the panoramic data is transferred to the device layer of the dispatch control center through the high-speed synchronous PTN (Packet Transport Network) network [18], [19]. The communication networking mode is essentially different from that of the current dispatching automation system, as shown in Fig. 2.

In Fig. 2, it is found that the structure of the communication system proposed in this paper is very different from that of the traditional power network communication system. In the equipment layer of the power grid dispatch control center, the panoramic data server executes the unified access and unified management of the steady, transient, dynamic and power billing data of the regional power grid. It will constitute a unified panoramic data platform. And the new panoramic data platform can provide support for applications of the power grid dispatch control center at all levels. This new type of communication transmission mode has the following advantages:

(1) Based on PTN communication technology, the unity of a variety of physical communication channel is realized. And the data transmission protocol is unified based on IEC61850 standard. The data access mode of the power grid dispatch control center is greatly simplified, and the data consistency is ensured too. At the same time, high speed real time PTN network fused with self-healing ring network technology has the very superior characteristics in network laying, expansion, online diagnosis and maintenance, etc.

(2) High speed synchronous PTN network can provide up to $2.5 \mathrm{G}$ bandwidth. And it is easy to achieve large capacity of network and high real-time transmission.

(3) In the DCIS based on RNCPM using IEEE1588 precise synchronous synchronization technology, it achieves the whole network data synchronization up to nanosecond level
[20], [21].

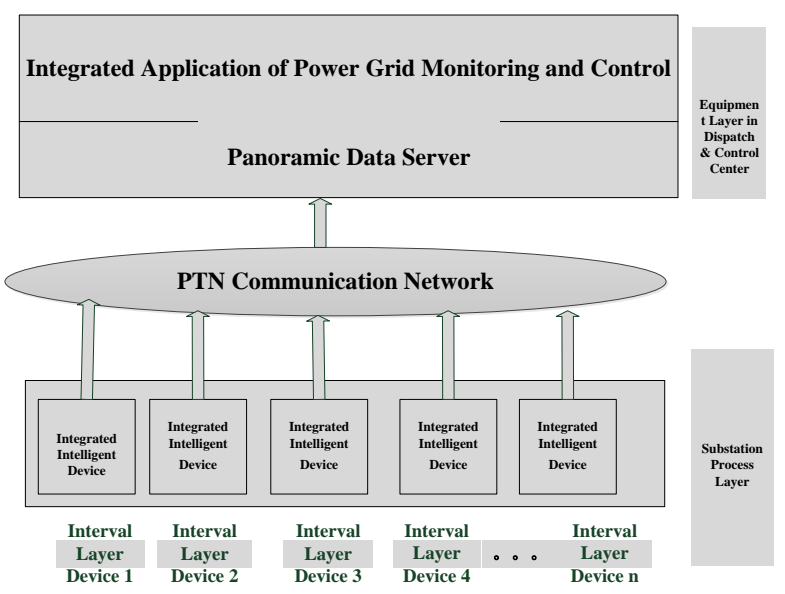

Fig. 2. Communication architecture of the DCIS based on RNCPM.

\section{The PANORAMic Data PlatForm}

\section{A. The Technology Architecture of the Panoramic Data Platform}

Under the framework of DCIS based on RNCPM, the regional network centralized panoramic data platform (RNCPDP) completes the data collection of steady state, transient state, dynamic state, electric quantities, by use of integrated intelligent equipment of the process layer. All the data is transmitted to the device layer of the power grid dispatch control center. Then, unified modeling and management of data based on IEC61850 standard are done in the RNCPDP. The platform system structure is shown in Fig. 3. The specific contents are as follows:

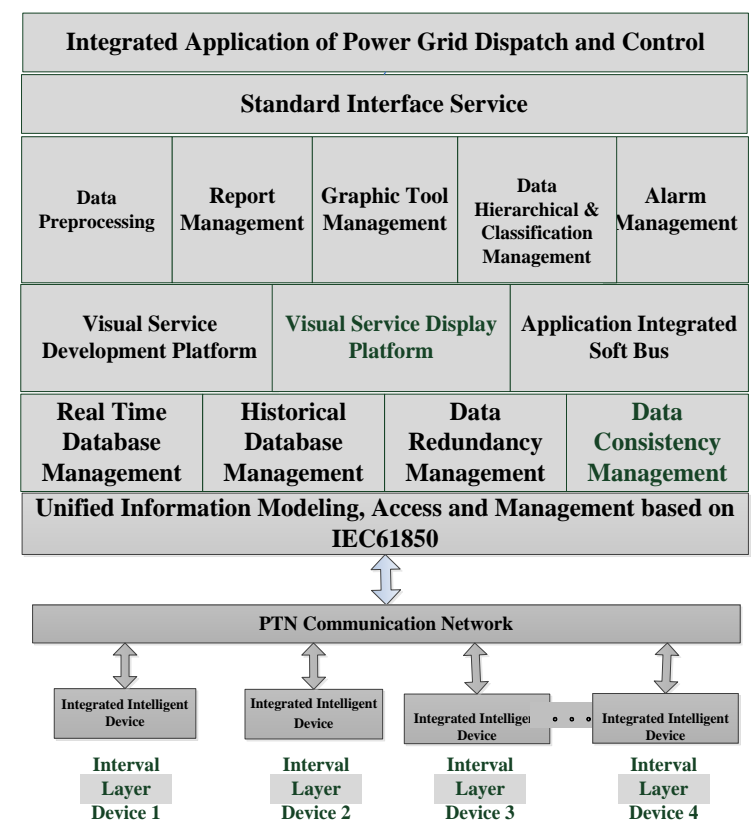

Fig. 3. Regional network protected panoramic data system platform.

\section{1) Data access layer}

The unified modeling and access of panoramic data is achieved in data access layer based on the ICE61850 standard.

The data access to panoramic data platform is as follows. 1) Status information of primary equipment, such as the state of switch or electric brake, alarm state of abnormal mechanism, 
etc. 2) Status information of secondary equipment, such as device self-test information, network communication abnormal alarm information, protection platen status information, etc. 3) Steady state data, such as current, voltage, active power, reactive power, frequency of power grid and so on. 4) Transient data, such as centralized protection action information and recorded information, etc. 5) Dynamic measurement of information, such as the current, voltage, active power, reactive power and phase angle with synchronous time scales. 6) Electric energy information, such as the active power, reactive power and time sharing statistical power.

\section{2) Data management layer}

Data management layer is the core function layer of the RNCPDP. It is responsible for the unified modeling of all the platform data. And other functions of the platform include the data identification preprocessing of all kinds of data, data hierarchical and classification processing, storage and processing of real time data and historical data, data redundancy and consistency management, data state exception alarm processing.

\section{3) Data access interface layer}

The data access interface layer provides a series of data access interface based on the IEC61850 standard, which supports the power grid dispatch control center to implement all levels of applications.

\section{B. Implementation Principle of Panoramic Data Platform}

\section{1) Implementation principle of data access layer}

The data access layer is implemented based on CSF (Communication Service Framework) [22], [23], and its basic schematic diagram is shown in Fig. 4. The specific contents are as follows:

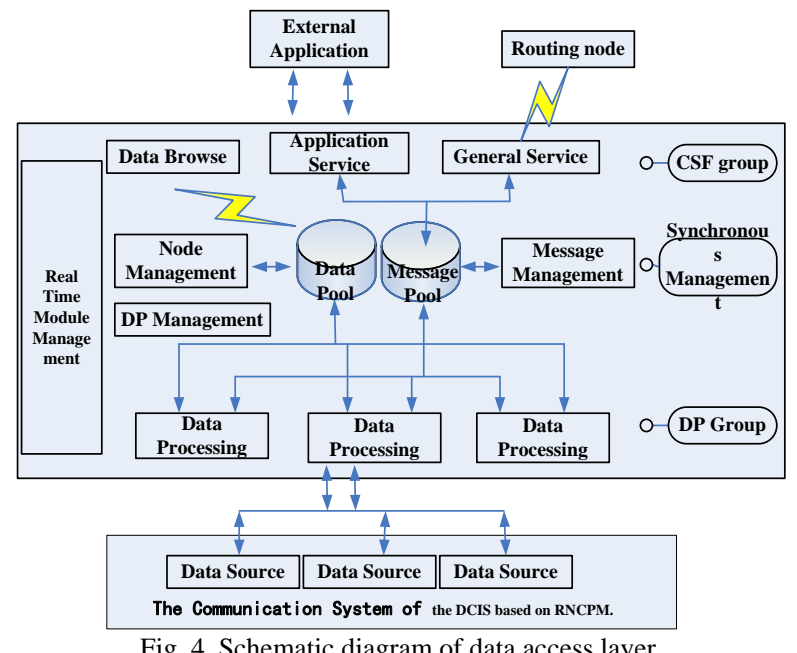

The IEC61850 model is established in the data access layer, which supports the mixed mode access with multiple protocols of the external data. When the external data source uses the IEC61850 protocol, seamless access can be achieved. The data access layer ensures the reliability and real time of access data by using of the priority packet pool and data pool. The CSF supports packet redundancy configuration management of communication services to meet the real-time and reliability requirements of massive data access. At the same time, the CSF provides data package, message compression, message encryption, identity authentication and so on to improve the safety and efficiency of data in the data transmission process.

The data access layer provides powerful debugging, diagnostic and monitoring tools for data source access. And It can realize the on-line monitoring and off-line analysis of original message of the access data, real-time data of data pool, communication node status and so on.

2) Implementation principle of data management layer

The data management layer is designed and implemented with the idea of hierarchical design based on the software platform of large-scale real-time control system. And the basic structure is as shown in Fig. 5.

It can be found from the Fig. 5 that the system is divided into three levels: the basic technology platform level, the basic application platform level and the platform application level. The specific contents are as follows.

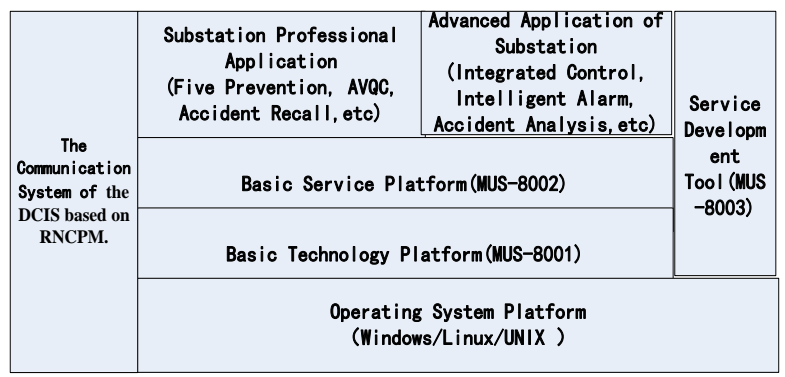

Fig. 5. Schematic diagram of data management layer.

The basic technology platform is responsible for the implementation of the system software architecture, shielding the operating system and various $\mathrm{I} / \mathrm{O}$ driver associated technology. The platform can realize the basic technical service function that has nothing to do with specific business. The following functions are included.

1)Object oriented unified data modeling management.

2)Distributed massive real-time database management.

3)Redundancy and consistency management of the real time and historical data

4)System message bus management.

5)Distributed process management.

6)Distributed privilege management.

7)Distributed log management.

8)Distributed resource management.

The basic application platform is business oriented and driven, and can quickly build application software. It is the core of the panoramic data platform software. Compared with the operating system platform and basic technology platform, the correlation between basic application platform and the user management and businesses is great. And it is a common platform for application software development, which solves the "interaction and management between the business description of application software to operating system platform and the basic technology platform ". The basic application platform includes the following functions.

1)Visualization business logic development.

2)Visualization service display.

3)Alarm service management.

4)Control service management.

5)Business object access management.

6)Data storage management.

7)Data forwarding service management.

Platform application level contains a series of professional 
applications based on the support of the system software platform, which realizes the panoramic data platform management function. The typical applications are as follows.

1)Data recognition processing.

2)Information stratification and classification processing.

3)Data state abnormal alarm processing.

4)Steady state data processing.

5)Transient data processing.

6)Dynamic data processing.

7)Power data processing.

3) Implementation principle of data access interface layer

The schematic diagram of the data access interface layer is shown in Fig 6. The specific contents are as follows.

Standard interface service provides IEC61850 standard service and IEC61970 CIM/CIS interface specification service for the power grid dispatch control center. The power grid dispatch control center class infrastructure applications and intelligent advanced applications can be realized based on the panoramic data provided by these two types of interface services to complete specific application functions. The main functions provided by the interface are as follows.

1)IEC61850 object model access.

2)ACSI abstract communication service.

3)GOOSE communication service.

4)IEC61850 SCL file parsing.

5)MMS communication service.

6)Mapping from IEC61850 SCL to IEC61970 CIM model. 7)IEC61970 CIM/CIS access service.

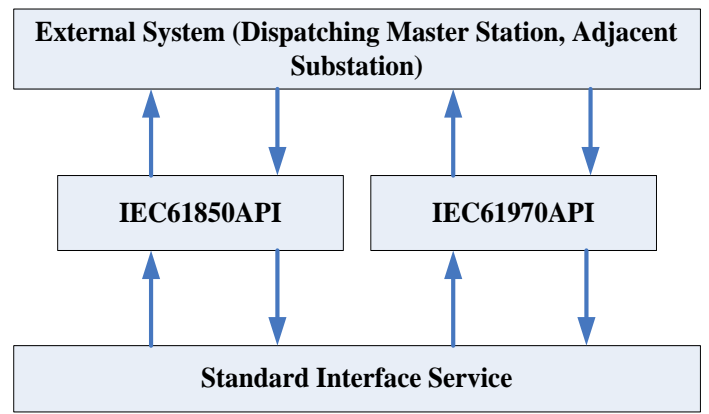

Fig. 6. Schematic diagram of data access interface layer.

\section{NeW FEATURES OF UNIFIED SECTION PANORAMIC DATA BASED ON RNCPM}

AS the DCIS based on RNCPM adopts a new communication system [22], the data uploaded to the control center will have the same identity, high real-time and accurate synchronization. The panoramic data of steady state, transient state, dynamic state and electric power in DCIS based on RNCPM will have the whole network unified cross section, which will have a profound impact on DCIS.

\section{A. The Influence of Steady State Data on DCIS}

The real-time index of the steady state data provided by the current substation monitoring system to DCIS is only 3-5 seconds. And the steady state data does not contain a synchronization time tag. This steady state data is usually applied in applications with low real-time and synchronization requirements of DCIS, such as, steady state power flow monitoring, real time data statistics, and historical report processing.

DCIS cancels the substation layer. And the integrated intelligent device of process layer transfers the steady data to the power grid dispatch control center through the high-speed PTN network directly. The real-time index can reach Millisecond level with the whole network time synchronization label. Because the integrity, real-time and synchronization of the information are ensured, in DCIS, it can realize the whole network unified section, visualization monitoring of synchronized power flow, real-time load forecasting and other application functions.

\section{B. Influence of Transient Data on DCIS}

The transient data provided by the current substation fault information system to the dispatch control center includes protection action information and fault record information. The transient data are generated by protection devices in substation interval. Because this kind of transient data contains only protection action and fault record information in this interval, accurate fault location and fault analysis cannot be realized [23].

In DCIS based on RNCPM, the integrated intelligent device in process layer uploads the original sampling message directly to the equipment layer of the dispatch control center through the high-speed PTN network. RNCPM is used to realize the protection function of regional power network, and the integrated fault reporting and recording information are generated at the same time. Because The integrated fault reporting and recording information generated by the RNCPM synthesizes the information of the whole network, accurate fault location and fault analysis can be carried out.

\section{Influence of Dynamic Data on DCIS}

The current substation uses the interval PMU (Phasor Measurement Unit) device to complete the data acquisition and processing of the dynamic data. And the dynamic data is uploaded to the WAMS ( Wide Area Measurement System ) of the dispatch control center by use of the station end PMU concentrator [24], [25]. But subject to the current mode of acquisition and processing of the substation information, the information transmission capacity and the cost of PMU device, PUM devices was sited and only built in hub lines and gateway points of important plant stations. The synchronous real-time measurement information provided to WAMS master station is very limited, and other measurement information still needs to be calculated by state estimation. On the one hand, it reduces the real-time performance of dynamic analysis, on the other hand, it affects the accuracy of analysis decision. Therefore, at present, the WAMS system can only realize the functions of dynamic monitoring, analysis and evaluation, which can only provide power grid security early warning and auxiliary decision-making information. But the real-time control cannot be achieved based on the real-time dynamic analysis results.

The DCIS has changed the traditional information collection and processing mode of substation automation system. The precise unification of the whole network clock is realized based on the IEEE1588 technology, which lays the foundation for the synchronous acquisition of information in each substation. The sampling value SV, GOOSE and 
synchronous phasor data with unified clock source are implemented in the substation process layer. The data are uploaded directly to the dispatch control center through the high-speed communication network. It provides data support for the application of synchronous data monitoring, analysis and control decision based on the whole network unified section for the dispatch control center. The whole network real-time synchronization information with the characteristic of the uniform cross section, information integrity and high real-time. Without state estimation, dynamic behavior analysis of power grid can be carried out, and fast decision-making and corresponding control strategy can be implemented. The control information transmission based on GOOSE guarantees the reliability and real-time of the real-time control of the power grid. DCIS based on RNCPM raises the power grid safety, stable operation ability to a new level.

\section{Influence of Electricity Data on DCIS}

The electric quantity information of the current substation automation system is collected and uploaded to the control center by the electric energy information collection terminal. The real-time monitoring and analysis of power cannot be carried out due to the low real-time and non-synchronization of power information.

In DCIS based on RNCPM, the electric energy information is collected and processed by integrated intelligent device in the process layer, which has high real-time performance and whole network synchronization characteristics. It can be used for real-time network loss calculation and analysis, providing technical support for realizing the economic operation of power grid and optimizing the operation mode of dispatching. And it can provide technical support for the economic operation of power grid and optimization of dispatching operation mode.

\section{ANALYSIS OF DISPATCHING OPERATION MANAGEMENT OF DCIS BASED ON RNCPM}

In DCIS based on RNCPM, the function of the substation control layer is moved up to the area monitor background of the control layer. The main and post protection of regional power grid is concentrated in the protection device of protective layer. The integrated design of control integration and regional monitoring background is successfully implemented [7]. The DCIS based on RNCPM will lead to substation equipment localization, integration of protection and monitoring, integration of control and monitoring, intelligent operation of power grid. This will revolutionize the dispatching operation management of DCIS [7], [8]. In the future smart grid, the intelligent dispatch control center will realize the power dispatching, management and operation of highly informative, intelligent, integrated and automated, which will become the command center of smart grid.

\section{A. High Degree of Unity of the Scheduling, \\ Communication, Automation and Protection of the Power Grid}

Because the existing dispatching automation system is set up with centralized control system and dispatching system, the following shortcomings are existed. First, centralized control system and dispatching system need to be built and maintained separately. Second, with the rapid development of power grid, the number of centralized control centers is growing rapidly. Because each centralized control center should be equipped with complete operation and maintenance personnel, the target to increase efficiency and improve the production efficiency cannot be achieved. Third, with the research and application of smart grid gradually, according to the traditional mode of operation and management, the division of the relay protection, communication, automation and other professional has not adapted to the development of technology, and the professional integration must be carried out to achieve the intensive, standardization of the personnel management. Fourth, with the further development of power grid and the continuous shortage of operators, the work intensity of centralized control in different periods is very different, and the problem of low operating efficiency continues to increase. Fifth, the intensification of production organization, the degree of specialization is not high. The chain of management is too long. The problem of low efficiency of resource allocation is gradually emerging. Above all, the traditional management mode of substation operation has been unable to meet the needs of the rapid development of modern power grid.

DCIS based on RNCPM realizes the unified modeling, unified collection, unified transmission and unified management of the data required by each professional application. It will lay a solid technical foundation for the realization of highly integrated unity of scheduling, communication, automation, protection and other professional. Through the intensive integration of power grid scheduling and equipment operation monitoring function, it has the following important significance.

1) it is beneficial to relieve the tension of personnel, reduce the number of substation operators, improve the efficiency of human resources, and reduce the workload of operators.

2) It will simplify the organization, optimize the allocation of the company's maintenance, operation and maintenance resources, adjust professional configuration, realize the professional management, and improve the management level.

3) It is helpful to improve the level of automation control and realize the intelligent operation monitoring.

\section{B. The Unique Data Source Ensures Data Consistency}

Because the data required for the application of professional control integration system respectively comes from different systems, such as the substation SCADA system, substation fault information system, electricity billing system and PMU system. it is very difficult to ensure the uniqueness and consistency of the data. The construction and maintenance of each independent system need to be made by different professionals, and the personnel cost and operation management cost are increased. At the same time, because the data source is not unique, it will affect the accuracy of the information analysis and processing of the integrated control system.

At the source of information, it realizes the integration and unification of the data required by various professional 
applications in DCIS based on RNCPM. Data transmission is carried out by using a unified network, and it ensures the uniqueness and consistency of the data sources in DCIS. The consistency of panoramic monitoring for real time operation of power grid is realized. It can also realize the unified management and unified maintenance of the professional application in DCIS.

\section{Unified Data Model is Used to Realize High Information Sharing}

The data needed for the application of the current DCIS are derived from different systems, and different data models and data transmission protocols are used to transmit them. At the same time, different transmission channels need to be set for different protocols for different transmission modes, which will lead to a variety of data access, exchange mode. It is difficult to realize high information sharing.

The unified modeling and unified management of steady-state, transient, dynamic and electrical data are realized based on the IEC61850 standard in the equipment layer of the dispatch control center. It provides a unified interface access mode for various applications, and realizes the highly integration and highly sharing of the whole network information.

\section{ADVANCED APPLICATION OF DCIS BASED ON RNCPM}

Transient data and dynamic data can be obtained through high-speed and real-time acquisition in the whole network. And the data is transmitted to DCIS through the high-speed communication network. Thus, the real-time monitoring and analysis calculation of the global normal operation and accident disturbance of the power grid is provided, and the dynamic process of the overall operation of the power grid is acquired and mastered in time. Furthermore, the automation and intelligent dispatching of the power grid can be realized. Fig. 7 is the architecture of intelligent dispatch control of power grid based on centralized network protection mode. And the specific contents are as follows. The intelligent scheduling with high real-time and centralized network protection mode will have a wide range of applications in the following aspects, such as intelligent control of power grid and self-healing of power grid, intelligent fault analysis and accident inversion simulation of power grid, regional protection and control of power grid, auxiliary decision-making of intelligent online dispatching of regional power grid, intelligent load rejection of power grid, etc.

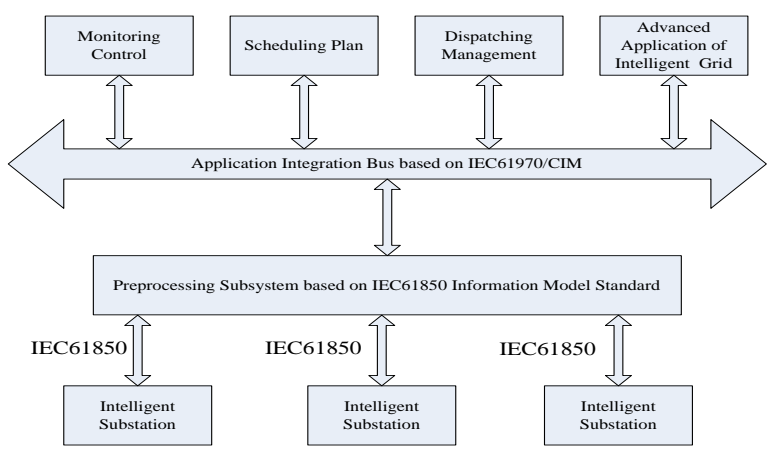

Fig. 7. System architecture of regional network protected dispatch control integration system.

\section{A. Intelligent Control and Self-Healing of Power Grid}

Intelligent control is the intelligent and automatic control function of processing operations of substation switching operation and normal state power grid accident exception recovery by use of the network topology analysis technology, programmable control technology and intelligent operation technology. The structure of intelligent control system of DCIS based on NCPM is shown in Fig. 8.

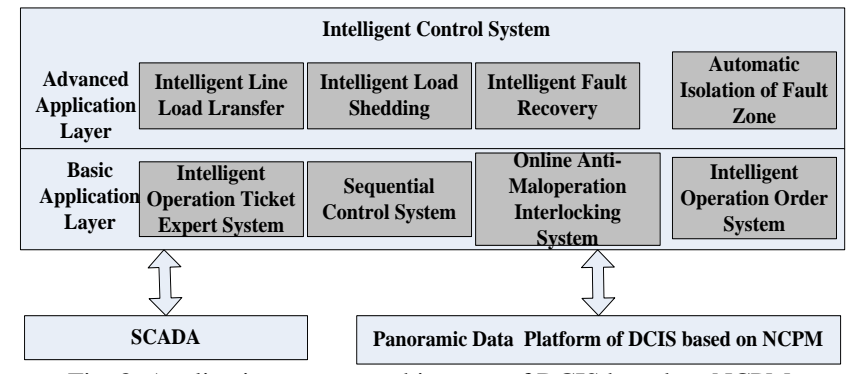

Fig. 8. Application system architecture of DCIS based on NCPM.

\section{B. Application of Intelligent Operation Ticket Technology and Intelligent Fault Recovery Operation Ticket in Regional Power Grid}

The main structure of the automatic recovery operation ticket is shown in Fig. 9. The specific contents are as follows.

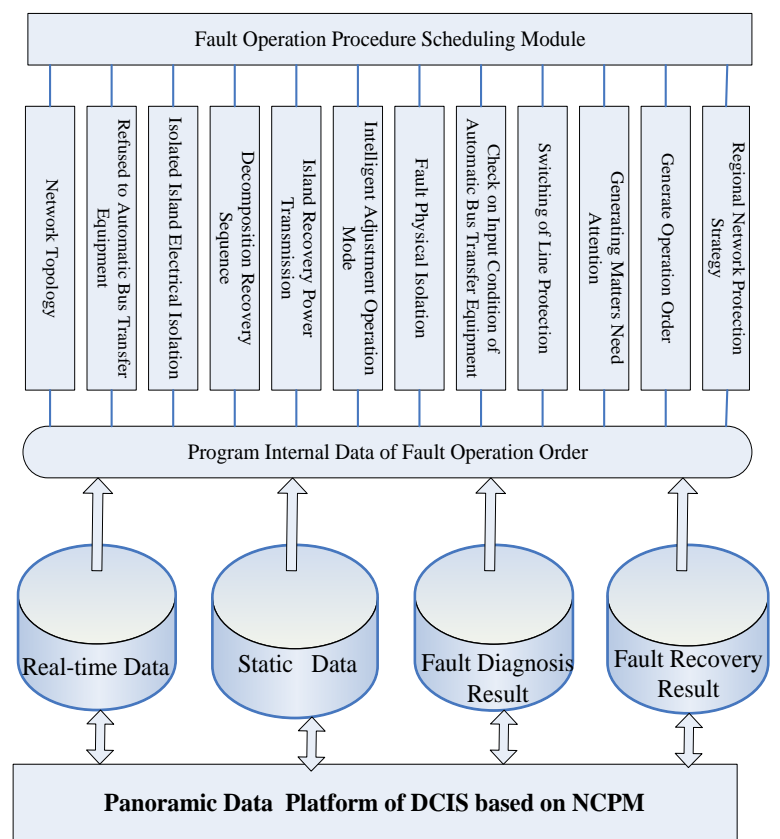

Fig. 9. Composition structure of smart grid automatic recovery operation order.

In Fig. 9, it can be found that the power grid fault operation order system is based on the SCADA system. According to the result of the fault recovery, the dispatching operation ticket is automatically generated under the condition of fault handling. The system has the following functions. The user can format the operation ticket based on the description of operation terms. The system can be started by the fault recovery program, or manually started. It can handle the operation sequence of the primary equipment under different wiring forms and adjust the system operation mode. The Electrical isolation operation of load island and fault equipment can be carried out. 


\section{CONCLUSION}

DCIS based on NCPM by using optical fiber communication technology, computer technology, sensor technology, relay protection and control of power grid operation, advanced technology, based on the IEC61850 standard, which is extended to scheduling, the scheduling within the scope of protection of centralized configuration, realize the integration of protection and control of power grid dispatching automation. The DCIS adopts platform + service support technology framework, which has the characteristics of system platform standardization, high integration and application intelligence. In the smart grid in the future, the management and operation of DCIS based on NCPM will achieve power scheduling, a high degree of information, intelligence, integration and automation. It is the command decision center of smart grid. Construction of all kinds of advanced applications for smart grid is in line with the research and development direction of DCIS on this basis of this system. The next phase of the project team will carry out the research on the power of things network and the intelligent city power application based on the model of this system.

\section{REFERENCES}

[1] T. G. Walmsley, R. W. M. Walmsley, and M. J. Atkins, "Energy return on energy and carbon investment of wind energy farms: A case study of New Zealand," Journal of Cleaner Production, vol. 167, pp. 885-895, November 2017.

[2] S. Kosunalp. "An energy prediction algorithm for wind-powered wireless sensor networks with energy harvesting," Energy, vol. 139, pp. 1275-1280, November 2017.

[3] H. Farokhi and M. H. Ghayesh, "Electrically actuated MEMS resonators: Effects of fringing field and nonlinear viscoelasticity," Mechanical Systems and Signal Processing, vol. 95, pp. 345-362, October 2017.

[4] A. Grantham, P. Pudney, L. A. Ward, D. Whaley, and J. Boland. "The viability of electrical energy storage for low-energy households," Solar Energy, vol.155, pp. 1216-1224, October 2017.

[5] L. Guo and F. Ding, "Least squares based iterative algorithm for pseudo-linear autoregressive moving average systems using the data filtering technique," Journal of the Franklin Institute, vol. 352, no. 10, pp. 4339-4353, October 2015.

[6] A. K. Rohit and S. Rangnekar. "An overview of energy storage and its importance in Indian renewable energy sector: Part II - Energy storage applications, benefits and market potential," Journal of Energy Storage, vol. 13, pp. 447-456, October 2017.

[7] H. X. Li, Y. Chen, M. Gül, H. Yu, and M. Al-Hussein. "Energy performance and the discrepancy of multiple NetZero energy homes (NZEHs) in cold regions," Journal of Cleaner Production, vol. 172, pp. 106-118, January 2018.

[8] P. Zhang, J. Chang, B. Qu, and Q. Zhao. "Denoising and trend terms elimination algorithm of accelerometer signals," Mathematical Problems in Engineering, vol. 2016, pp. 1-9, April 2016.

[9] B. Keitt, R. Griffiths, S. Boudjelas, et al., "Best practice guidelines for rat eradication on tropical islands," Biological Conservation, vol. 185, pp. 17-26, April 2015.

[10] P. Millan, C. Molina, E. Medina, et al., "Time series analysis to predict link quality of wireless community networks," Computer Networks, vol. 93, part 2, pp. 342-358, 2015.
[11] B. Kok and H. Benli, "Energy diversity and nuclear energy for sustainable development in Turkey," Renewable Energy, vol. 111, pp. 870-877, October 2017.

[12] P. Peris-Lopez and H. Martin, "Hardware trojans against virtual keyboards on E-banking platforms - A proof of concept," AEU International Journal of Electronics and Communications, pp, 146-151, April 2017.

[13] S. Chandrasekhar, A. Ibrahim, and M. Singhal, "Renewable and sustainable energy reviews," Mechanical Systems and Signal Processing, vol. 67, pp. 73-88, June 2017.

[14] N. Vidadili, E. Suleymanov, C. Bulut, and C. Mahmudlu, "Transition to renewable energy and sustainable energy development in Azerbaijan," Renewable and Sustainable Energy Reviews, vol. 80, pp. 1153-1161, December 2017.

[15] Z-M. Sha, Y.-S. Hao, et al., "A new algorithm for PMU placement optimization in power system," RELAY, vol. 33, no. 7, pp. 31-36, July 2015.

[16] W. Cong, Z.-C. Pan, and J.-G. Zhao, "A wide area relaying protection algorithm based on longitudinal comparison principle," in Proc. the CSEE, December 2016, vol. 26, no. 21, pp. 8-14.

[17] A. Montazeri and C. J. Taylor, "Modeling and analysis of secondary sources coupling for active sound field reduction in confined spaces," Mechanical Systems and Signal Processing, vol. 95, pp. 286-309, October 2017.

[18] S. Tuohy, A. Winterlich, B. McGinley, M. Glavin, E. Jones, P. Denny, and L. Kilmartin, "Evaluating the influence of packet loss on visual quality of perception for high bandwidth automotive networks Original," Signal Processing: Image Communication, vol. 43, pp. 15-27, April 2016

[19] A. Silvast, "Energy, economics, and performativity: Reviewing theoretical advances in social studies of markets and energy," Energy Research \& Social Science, vol. 34, pp. 4-12, December 2017.

[20] G. Marinelli, M. Bassani, M. Piras, and A. M. Lingua, "Mobile mapping systems and spatial data collection strategies assessment in the identification of horizontal alignment of highways," Transportation Research Part C: Emerging Technologies, vol. 79, pp. 257-273, June 2017.

[21] K. Seddig, P. Jochem, and W. Fichtner, "Integrating renewable energy sources by electric vehicle fleets under uncertainty," Energy, vol. 141, pp. 2145-2153, December 2017.

[22] V. Mitra, G. Sivaraman, H. Nam, C. Espy-Wilson, E. Saltzman, and M. Tiede, "Hybrid convolutional neural networks for articulatory and acoustic information based speech recognition," Speech Communication, vol. 89, pp. 103-112, May 2017.

[23] S. L. Gay, A. Mille, O. L. Georgeon, and A. Dutech, "Autonomous construction and exploitation of a spatial memory by a self-motivated agent," Cognitive Systems Research, vol. 41, pp. 1-35, March 2017.

[24] Z.-G. Guo, J. Chen, J.-S. Chen, et al., "Discussion on some problems of large hydroelectric unit protection," Power System Protection and Control, vol. 39, no. 3, pp. 148-151, March 2011.

[25] Pushpkant and S. K. Jha. "Comparative study of different classical and modern control techniques for the position control of sophisticated mechatronic system," Procedia Computer Science, vol. 93, pp. 1038-1045, 2016.

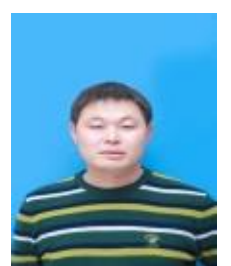

Zhang Peng was born in the village of Qixian County, Henan Province, China, on February 6, 1979. He received his M.Sc \& Ph.D from the Automation College of Northwestern Polytechnical University in Xi'an China. He is currently a professor in the information science and electrical engineering department at Zhongyuan University of Technology, China. He is also affiliated with HaMi Technical College, China, as an adjunct faculty staff. 